\title{
Simulation of turbulent explosion of hydrogen-air mixtures
}

\author{
I. Ahmed, N. Swaminathan* \\ Department of Engineering, University of Cambridge, Cambridge, CB2 1PZ, UK
}

\begin{abstract}
Spherically expanding turbulent premixed hydrogen-air flames are computed using the Reynolds-Averaged Navier-Stokes (RANS) approach. The mean reaction rate is modelled using unstrained and strained flamelets, and an algebraic model. Since the temperature and mass fraction evolve differently in hydrogen flames because of non-unity Lewis numbers, two reaction progress variables are used in the calculations. The computed turbulent burning velocity is compared to measured values to validate the computational models. The strained flamelets model captured the experimental variations quite well while the other two models over estimated the burning velocity of stoichiometric hydrogen-air flames. All of these models under estimated the burning velocity for a lean flame which is thermo-diffusively unstable, indicating a need to include these instability effects in turbulent combustion modelling. A comparative analyses of stoichiometric hydrogen- and methane-air flames are also performed to understand their relative behaviour for a given turbulent combustion condition signified by $u^{\prime} / s_{L}^{0}$ and $\Lambda / \delta$. Although the gross behaviour is observed to be similar, there is a significant difference in the normalised turbulent flame speeds, $s_{t} / s_{L}^{0}$, of these flames. A detailed analysis showed that the mean reaction rate increases very sharply, at least by two order of magnitude, near the leading edge for the hydrogen flames compared to the methane flames. Since this behaviour is controlled not only by turbulence and its interaction with flame but also by chemical kinetics, one must also consider chemical kinetics parameters such as activation temperature, in addition to $u^{\prime} / s_{L}^{0}$ and $\Lambda / \delta$ to characterise $s_{t} / s_{L}^{0}$.
\end{abstract}

\footnotetext{
${ }^{*}$ Corresponding author. Tel. +44 1223332586

E-mail address: ns341@cam.ac.uk (N. Swaminathan)
} 
Keywords: hydrogen, flamelet model, turbulent premixed, spherical flame

\section{Introduction}

The envisaged depletion of fossil fuel resources and a need to reduce pollutants emission from combustion have led to a surge in finding alternative energy sources. Hydrogen is considered as a potential future energy carrier with many benefits over the current hydrocarbon fuels [1-3]. In particular, good combustion characteristics of hydrogen make it an attractive fuel for internal combustion engines $[4,5]$. Hydrogen has certain favourable combustion properties such as wide flammable range and large burning velocity, which render hydrogen as an ideal additive to improve combustion characteristics of new and bio-derived hydrocarbon fuels [6]. Also, fundamental understanding of hydrogen combustion is important from safety view points, for example generation and accumulation of hydrogen in nuclear reactors [7] and rupturing of a pressurised hydrogen storage tank can lead to explosions.

A spherically expanding flame is commonly used to investigate fundamental characteristics of hydrogen-air combustion from various view points and these studies related to internal combustion engines [5] and safety aspects $[8,9]$ have been reviewed in the past. This flame configuration has also been used to measure laminar burning velocity, $s_{L}^{0}$, and the influence of fluid dynamic stretch, $\kappa$, on flame propagation has been ignored in some of those earlier studies. This influence is typically given by $s_{L}=s_{L}^{0}-\kappa \mathcal{L}$ for small values of $\kappa$ and the Markstein length scale $\mathcal{L}$ can be positive or negative [10]. The stretch rate is defined as the rate of change of flame area per unit area, $A$, ie., $\kappa=(d A / d t) / A$ and it is given as $\kappa=\left(2 / r_{f}\right)\left(\mathrm{d} r_{f} / \mathrm{d} t\right)$, for a spherically expanding flame of radius $r_{f}$ [10]. Later studies [11-18] showed that the stretch effects must be included in the analysis to explain the presence of cellular instabilities observed in experiments of lean hydrogen-air spherical flames. The additional flame area resulting from this instability led to an increase in $s_{L}$ implying a negative $\mathcal{L}$ for thermo-diffusively unstable lean hydrogen-air mixtures. The stoichiometric and rich mixtures showed positive $\mathcal{L}$.

Since the thermo-diffusive instabilities result from differential and/or preferential diffusion phenomena, the Lewis number, defined as the ratio of thermal to mass diffusivity of a deficient reactant in the mixture, is typically used to identify thermo-diffusively unstable mixtures. Lewis number is typically 
less than unity for lean hydrogen-air mixtures [10] and these flames are more susceptible to cellular instability [15, 18-20]. A review of these studies on thermo-diffusive instabilities can be found in [21].

Turbulent spherical hydrogen-air flames have been investigated using fanstirred bombs [20, 22-25] and wind tunnels with grid turbulence $[26,27]$ to address the role of turbulence since practical combustion invariably includes turbulence. These studies showed that the turbulent burning velocity is increased when the reactant mixture yields thermo-diffusively unstable flames and this effect is pronounced when the turbulence is weak. Accounting for thermo-diffusive instability effects in turbulent combustion modelling is a challenging task and is still an open question although some attempts has been made in the past to include hydrodynamic instability effects [28]. One way to account for thermo-diffusive instability effects is to use an effective Lewis number to modify the turbulent burning velocity expressions as has been done in [29] for hydrocarbon flames. An alternative approach is to include the instability effects in laminar flame speed correlations obtained using spherically expanding laminar flames and use them as input to turbulent combustion models based on turbulent burning velocity or flame surface density [30]. These approaches were shown to yield a satisfactory comparison with measurements for laboratory scale flames [29], a single-cylinder compression machine [30] and spark-ignited internal combustion engines [31]. Following the second approach, the thermo-diffusive effects are expected to be included when $s_{L}^{0}$ calculated with detailed chemistry and transport is used and this philosophy of including thermo-diffusive effects, however, does not seem to be adequate for a test case considered here as one shall see later in section 4.1 .

In this work, Reynolds-Averaged Navier Stokes (RANS) methodology is used to simulate spherically exploding turbulent flames of hydrogen-air mixtures. The turbulence-chemistry interaction is modelled using strained flamelets model [32], which has been used to study spherical turbulent methaneair flames [33]. The specific objectives of this work are:

1. To assess the strained flamelets model for turbulent spherical hydrogenair flames by comparing measured [20] and computed results;

2. To compare and contrast turbulent spherical flame propagation characteristics of hydrogen- and methane-air mixtures having the same equivalence ratio and turbulence conditions.

The performance of strained flamelets model is also assessed here in compar- 
ison to other flamelets based models available in the literature.

This paper is organised as follows. The numerical problem setup is described in the next section. The test cases used for model validation are described in Section 3. The numerical simulation results are discussed in Section 4 along with a comparison of various reaction rate closures. The conclusions are summarised in the final section.

\section{Numerical setup}

The radially expanding spherical turbulent hydrogen-air flames are simulated using the unsteady RANS (URANS) approach. These flames are assumed to be spherically symmetric and thus only the radial terms of the governing equations, which are written in spherical coordinates, are retained for simulations. These are Favre-averaged conservation equations for the mass, radial momentum, turbulent kinetic energy, $\widetilde{k}$, and its dissipation rate, $\widetilde{\varepsilon}$, a reaction progress variable, $\widetilde{c}$, and its variance, $\sigma_{c}^{2}$. The explicit form of these equations are given in [33] and the contributions of the centrifugal forces per unit volume arising from the Reynolds stresses in the angular $(\theta)$ and azimuthal $(\phi)$ directions of a spherical systems are included appropriately for the radial momentum, $\widetilde{k}$ and $\widetilde{\varepsilon}$ equations. The turbulent stresses and fluxes are modelled using eddy diffusivity hypothesis as in [33]. The contribution of chemical reactions appear as a mean reaction rate, $\overline{\dot{\omega}}$, in $\widetilde{c}$ equation and as $\overline{\dot{\omega}^{\prime \prime} c^{\prime \prime}}$ for $\sigma_{c}^{2}$ equation. The effect of combustion on other conservation equations is felt through a change in the mean density of the fluid mixture as has been shown in [33] and the mean density is calculated using the equation of state.

\subsection{Reaction rate modelling}

The mean reaction rate, $\overline{\dot{\omega}}$, is modelled using the strained flamelet model [32], which is described briefly below. The laminar premixed flames established in counter flowing streams of reactant and product are used as model flames in this approach and the mean reaction rate is given by $[32,33]$

$$
\overline{\dot{\omega}}=\int_{0}^{1}\left[\int_{N_{1}}^{N_{2}} \dot{\omega}(\zeta, \psi) P(\psi \mid \zeta) \mathrm{d} \psi\right] P(\zeta) \mathrm{d} \zeta,
$$

where $\zeta$ and $\psi$ are sample space variables respectively for $c$ and instantaneous scalar dissipation rate, $N=\mathcal{D}(\nabla c \cdot \nabla c)$, where $\mathcal{D}$ is the molecular diffusivity 
of $c$. The flamelet reaction rate, $\dot{\omega}(\zeta, \psi)$, is obtained from the laminar flames and the integration limits $N_{1}$ and $N_{2}$ are obtained using results of fully burning and almost extinguished flamelets [32].

A comprehensive chemical kinetics mechanism is used in the laminar flame calculations and a number of such mechanisms are available for hydrogenair combustion. Figure 1 compares unstretched laminar burning velocity, $s_{L}^{0}$, computed in this study employing several such mechanisms [34-38], denoted as MECH in Fig. 1, with measured values available in the open literature [11, 14, 15, 18, 20, 39-48]. These experiments, denoted as EXP in Fig. 1, were carried out at atmospheric pressure and reactant temperature of about $300 \mathrm{~K}$ using various flame configurations such as outwardly propagating spherical, stagnation point and counter-flow flames. These results are shown in Fig. 1 only for the equivalence ratio spanning from lean to stoichiometric range. There is a large scatter in the experimental data and the computational results for various chemical mechanisms used here agree quite well for lean mixtures but the values of $s_{L}^{0}$ obtained using the mechanisms of Westbrooke et al. [34] and Miller et al. [35] are beyond the experimental scatter. The values obtained using the mechanisms of Ó Conaire et al. [36], Li et al. [37] and Burke et al. [38] are in good agreement with the experimental data for $\phi=1$ mixture. The mechanism of Li et al. [37] is used in this study to calculate turbulent spherical flames of hydrogen-air mixture having $\phi=0.4$ and 1 .

The presumed pdfs, $P(\zeta)$ and $P(\psi \mid \zeta)$, are specified using the $\beta$ and lognormal functions respectively [32]. The $\beta$ function requires $\tilde{c}$ and $\sigma_{c}^{2}$, which are obtained by solving their transport equations. The lognormal function requires the conditional mean and variance of $\ln (N \mid \zeta)$, which are obtained as in [32]. The mean scalar dissipation rate, $\widetilde{\epsilon}_{c}$, required for $\sigma_{c}^{2}$ transport equation and the lognormal function need to be modelled. A number of models, as summarised in [49], are available for this quantity and a simple model [50] satisfying the realisability condition of $\widetilde{\epsilon}_{c} \geq 0$ is used in this study. This model is written as

$$
\widetilde{\epsilon}_{c} \simeq \frac{1}{\beta^{\prime}}\left[\left(2 K_{c}^{*}-\tau C_{4}\right) \frac{s_{L}^{0}}{\delta_{L}^{0}}+C_{3} \frac{\widetilde{\varepsilon}}{\widetilde{k}}\right] \sigma_{c}^{2},
$$

where $\delta_{L}^{0}$ is the unstrained laminar flame thermal thickness, $\tau$ is the heat release rate parameter, and $\widetilde{k}$ and $\widetilde{\varepsilon}$ are respectively the Favre-averaged turbulent kinetic energy and its dissipation rate. The various model parameters are: $\beta^{\prime}=6.7, C_{3}=1.5 \sqrt{\mathrm{Ka}} /(1+\sqrt{\mathrm{Ka}})$ and $C_{4}=1.1 /(1+\mathrm{Ka})^{0.4}$. The 


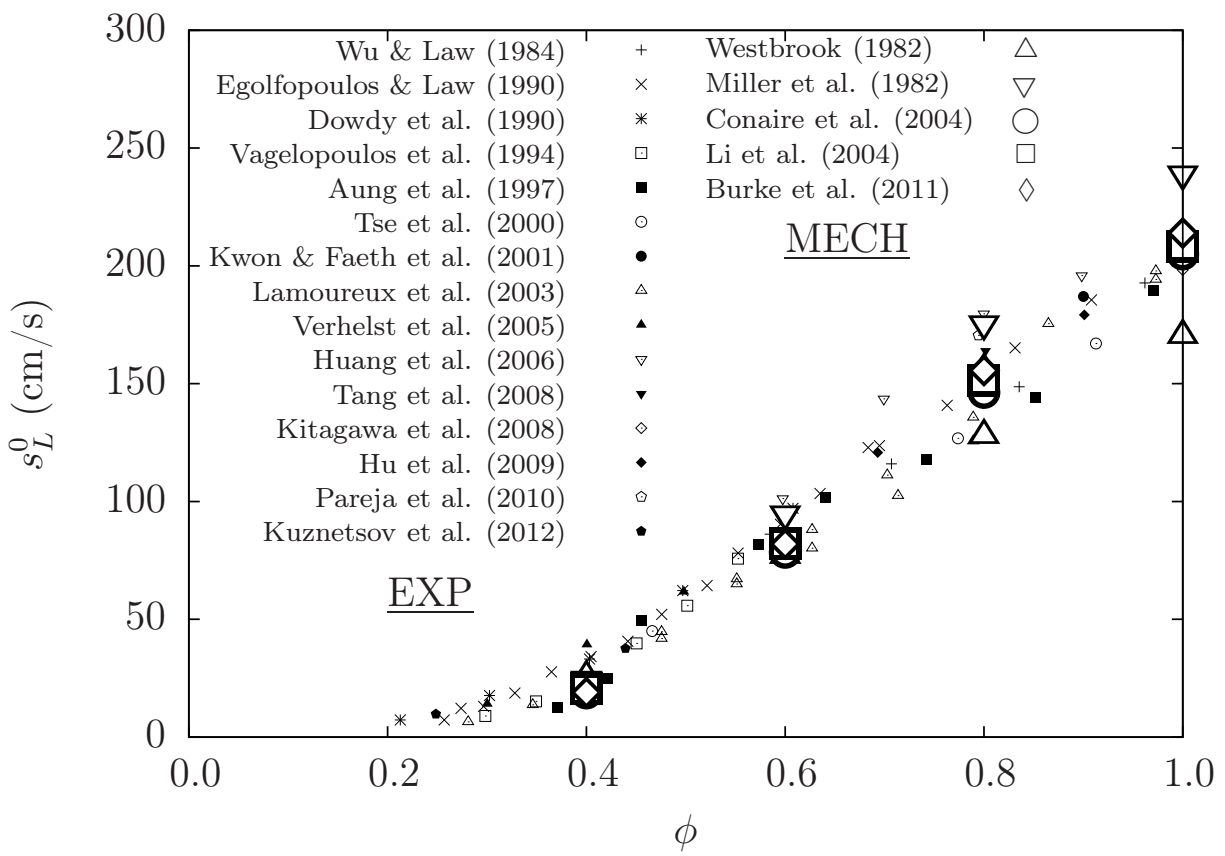

Fig. 1: Comparison of computed and measured unstretched laminar burning velocity for various equivalence ratios at atmospheric conditions. The experimental values are obtained from 15 earlier studies. 
parameter, $K_{c}^{*}$ takes a value of $0.73 \tau$ for $\phi=0.4$ and $0.66 \tau$ for $\phi=1.0$ hydrogen-air mixtures [51]. The above parameters are specified to satisfy certain physical aspects of turbulence-flame interaction $[50,52]$ and thus they cannot be changed arbitrarily. The Karlovitz number, Ka, is defined as $\mathrm{Ka} \equiv$ $\left(u^{\prime} / s_{L}^{0}\right)^{3 / 2}(\delta / \Lambda)^{1 / 2}$, where $u^{\prime}=(2 \widetilde{k} / 3)^{1 / 2}, \Lambda=u^{\prime 3} / \widetilde{\varepsilon}$ and $\delta=\delta_{L}^{0} /\left[2(1+\tau)^{0.7}\right]$. The model for $\widetilde{\epsilon}_{c}$ in Eq. (2) does not include the influence of mean curvature that might exists in spherical flames. However, correcting Eq. (2) for mean curvature effects did not show a significant difference in spherical flame propagation [33]. Thus, Eq. (2) is used for this study. For given values of control-variables, $\widetilde{c}, \sigma_{c}^{2}$ and $\widetilde{\epsilon}_{c}$, one builds a table for $\overline{\dot{\omega}}$ using Eq. (1). This look-up table is accessed during turbulent flame calculations to obtain the source terms for $\widetilde{c}$ and $\sigma_{c}^{2}$ equations.

These source terms related to chemical reactions are also closed using two other models for comparative analyses. First of these is an algebraic model [53]:

$$
\overline{\dot{\omega}}=\frac{2}{2 C_{m}-1} \bar{\rho} \widetilde{\varepsilon}_{c} \quad \text { and } \quad \overline{\dot{\omega}^{\prime \prime} c^{\prime \prime}}=\left(C_{m}-\widetilde{c}\right) \overline{\dot{\omega}},
$$

where values for $C_{m}$ are respectively 0.75 and 0.56 for $\phi=0.4$ and stoichiometric hydrogen-air mixtures [51]. This model for $\overline{\dot{\omega}}$ does not include chemical kinetic detail and Eq. (2) is used for $\widetilde{\epsilon}_{c}$. The second model uses unstrained laminar flames as flamelets and is given by

$$
\overline{\dot{\omega}}=\int_{0}^{1} \dot{\omega}_{o}(\zeta) P(\zeta) \mathrm{d} \zeta
$$

where $\dot{\omega}_{o}$ is the reaction rate of a planar unstrained laminar flame computed using detailed chemical kinetics as for the strained flamelets and the look-up table for this model has only $\widetilde{c}$ and $\sigma_{c}^{2}$ as the control-variables. These models have been used in the past to study statistically planar [32] and spherical [33] methane-air flames, which typically have unity Lewis number. Hydrogen-air flames have typically non-unity Lewis number and the method used in this study to account for this effect is described next.

\subsection{Accounting for non-unity Lewis number}

One needs at least two degrees of freedom to describe the thermo-chemical state of a mixture with non-unity Lewis number since the temperature and mass fraction evolve differently. Thus, two progress variables are used in this 
study to account for this. One is based on $\mathrm{H}_{2} \mathrm{O}$ mass fraction normalised by its burnt side value in an unstrained laminar flame, defined as $c=c_{\mathrm{H} 2 \mathrm{O}}=$ $Y_{\mathrm{H} 2 \mathrm{O}} / Y_{\mathrm{H} 2 \mathrm{O}}^{b}$ and another one is based on temperature $c_{T}=\left(T-T_{u}\right) /\left(T_{u}-T_{b}\right)$. The variation of $c$ with $c_{T}$ in the laminar strained and unstrained flames is shown in Fig. 2a. If $c$ and $c_{T}$ are identical then the variation in Fig. 2a should fall on the diagonal line and this result clearly show that the difference between these two progress variables is large on the burnt side. This is expected and a similar observation has been made in turbulent flames also [54]. The mixture molecular weight also changes considerably, nearly $18 \%$, across the flamelets as shown in Fig. 2b. For these reasons, the mean density is calculated as

$$
\frac{\bar{\rho}}{\rho_{u}}=\left(\frac{\bar{M}}{M_{u}}\right) \frac{1}{\left(1+\tau \widetilde{c}_{T}\right)}
$$

using the equation of state. The non-unity Lewis number effects on the chemical source terms is presumed to be included at the flamelets level since detailed transport and chemical kinetics are used to compute the necessary laminar flames. This is akin to the methodology used in earlier studies as noted in the introduction. Thus, two Favre-averaged progress variables, $\widetilde{c}$ and $\widetilde{c}_{T}$, and $\sigma_{c}^{2}$ equations in addition to other conservation equations are solved for the turbulent flames and their chemical source terms, $\overline{\dot{\omega}}_{c}, \overline{\dot{\omega}}_{c_{T}}$ and $\overline{\dot{\omega}}^{\prime \prime} c^{\prime \prime}$, are obtained from the look-up tables as described in section 2.1. Since the molecular diffusivities are smaller than turbulent diffusivities, the difference between the $\widetilde{c}$ and $\widetilde{c}_{T}$ transport equations is only in their respective source terms.

\subsection{Computational detail}

The flamelets for the look-up table construction are calculated using the PREMIX [55] and OPPDIF [56] codes. Detailed reaction mechanism of Li et al. [37] is used for combustion kinetics of hydrogen-air mixture. Note that thermal diffusion effects are included in these calculations.

The necessary conservation equations, along with the combustion models discussed in the previous two subsections are solved using the finite-volume methodology for turbulent spherical flames. Power law scheme [57] is used for convection-diffusion and an implicit first order backward Euler method is used for time stepping. The pressure-velocity coupling is through the SIMPLER approach [57]. 


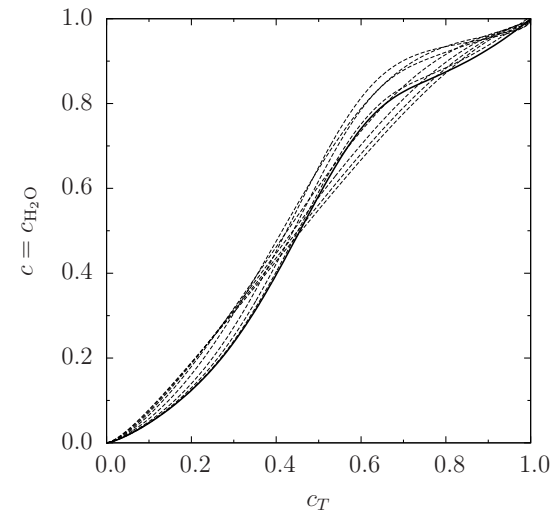

(a)

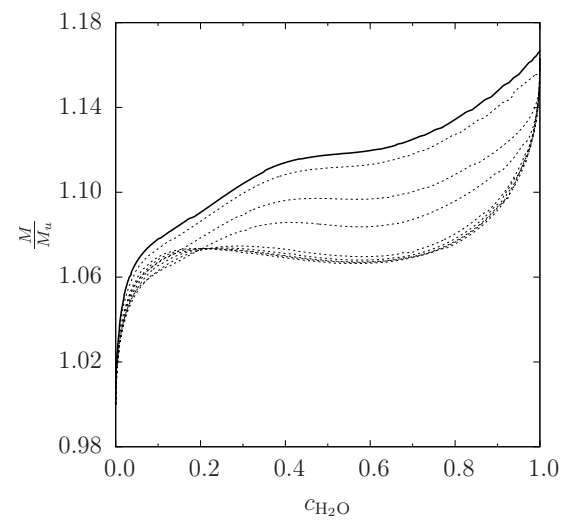

(b)

Fig. 2: Variation of (a) $c=c_{\mathrm{H}_{2} \mathrm{O}}$ with $c_{T}$ and (b) mixture molecular weight, $M$, normalised by its unburnt side value, across unstrained (solid line) and strained (dashed lines) flamelets.

The computational domain length is chosen so that the turbulent flame brush will remain inside the domain over the whole simulation period. Uniform grid-spacing is used ensuring that there are at least 10 grid points inside $\min \left(\Lambda, \delta_{t}\right)$, where the turbulent flame brush thickness is defined as $\delta_{t} \equiv 1 /|\partial \tilde{c} / \partial r|_{\max }$. The time step size is chosen to be $0.1 \mu$ s to ensures numerical stability and the resolution of reaction, diffusion and convection time scales on a chosen spatial grid for given turbulence and thermo-chemical conditions.

Initial values of $\widetilde{k}$ and $\widetilde{\epsilon}$ for the unburnt mixture are specified for the entire computational domain using the chosen value of $u^{\prime}$ and $\Lambda$. The initial spatial variation of $\widetilde{c}(r)$ and $\widetilde{c}_{T}(r)$ are chosen to be the same for the sake of simplicity. These initial variations have 0 in the unburnt and 1 in the burnt mixtures and is chosen after few tests to minimise the initial transients for given turbulence and thermo-chemical conditions. The initial values of $\widetilde{u}(r)$, $\bar{\rho}(r)$ and $\bar{p}(r)$ are specified to be consistent with the variations of $\widetilde{c}(r)$ and $\widetilde{c}_{T}(r)$. The following boundary conditions apply for a flame propagating radially outward in an unconfined domain:

$$
\widetilde{u}_{r}(0, t)=\left.\frac{\partial \tilde{c}}{\partial r}\right|_{(0, t)}=\left.\frac{\partial \tilde{k}}{\partial r}\right|_{(0, t)}=\left.\frac{\partial \tilde{\varepsilon}}{\partial r}\right|_{(0, t)}=\left.\frac{\partial \sigma_{c}^{2}}{\partial r}\right|_{(0, t)}=0 .
$$


For the unburnt mixture $\left(r=r_{1}\right)$

$$
\left.\frac{\partial \tilde{u}_{r}}{\partial r}\right|_{\left(r_{1}, t\right)}=\left.\frac{\partial \tilde{k}}{\partial r}\right|_{\left(r_{1}, t\right)}=\left.\frac{\partial \tilde{\varepsilon}}{\partial r}\right|_{\left(r_{1}, t\right)}=0, \quad \sigma_{c}^{2}\left(r_{1}, t\right)=\tilde{c}\left(r_{1}, t\right)=0, \quad \bar{p}\left(r_{1}, t\right)=p_{\infty} .
$$

\section{Test flames}

The numerical model of an outwardly propagating turbulent spherical flame described in the previous section is used to investigate turbulence effect on the propagation of hydrogen-air spherical flames. The results of these simulations will be compared to spherical methane-air flames studied in [33]. Before discussing these test cases, experimental flames used to assess the strained flamelets model for hydrogen-air mixture is described.

\subsection{Experimental flames}

Spherically expanding turbulent hydrogen-air flames inside a fan-stirred combustion vessel investigated by Kitagawa et al. [20] are considered for strained flamelets model assessment. This experimental study considered four different equivalence ratios $(0.4,0.6,0.8$ and 1.0) at three different initial pressures, 1, 2.5 and $5 \mathrm{~atm}$. and an initial temperature of $300 \mathrm{~K}$. Out of these cases, only the atmospheric flames with $\phi=0.4$ and 1 are considered here for computational reasons. Thermo-chemical characteristics of these two flames are given in Table 1 and $\tau \neq \rho_{u} / \rho_{b}-1$, specifically for $\phi=1$ case, due to the variation of mixture molecular weight across the flame front as noted earlier.

Two fans, continuously running during the experiments, were used to mix the reactants inside the vessel and to generate non-decaying isotropic turbulence. Experiments were conducted at two turbulence intensities and the higher turbulence case with r.m.s of velocity fluctuations, $u^{\prime}$, of $1.59 \mathrm{~m} / \mathrm{s}$ is simulated in this study. The experimentally determined turbulence integral length scale, $\Lambda$, was $10.3 \mathrm{~mm}$. Since the turbulence in the experiment is nondecaying, values of $\widetilde{k}$ and $\widetilde{\varepsilon}$ were frozen for the simulations. The propagation of turbulent flames was recorded using high speed schlieren photography in the experiments [20] and since schlieren images show the flame leading edge [58], the flame radius, $r_{f}$, was defined using $\widetilde{c}=0.05$ in the simulations. 
Table 1: Thermo-chemical conditions of experimental flames [20] considered for this study

\begin{tabular}{cccccc}
\hline$\phi$ & $s_{L}^{0}(\mathrm{~m} / \mathrm{s})$ & $\delta_{L}^{0}(\mathrm{~mm})$ & $\delta(\mathrm{mm})$ & $\rho_{u} / \rho_{b}$ & $\tau$ \\
\hline 0.4 & 0.21 & 0.67 & 0.09 & 4.31 & 3.66 \\
1.0 & 1.98 & 0.35 & 0.01 & 6.84 & 6.97 \\
\hline
\end{tabular}

\subsection{Test cases for further analyses}

Outwardly propagating turbulent spherical flames in an unconfined space are considered and the influences of heat release on turbulence are also included in these simulations by solving for $\widetilde{k}$ and $\widetilde{\varepsilon}$ unlike in the above experimental cases. Only stoichiometric hydrogen-air mixture at $300 \mathrm{~K}$ and atmospheric pressure are considered. The hydrogen-air flame results are to be compared with stoichiometric methane-air flames under the same initial pressure, temperature, and turbulence $\left(u^{\prime} / s_{L}^{0}\right.$ and $\left.\Lambda / \delta\right)$ conditions to understand their relative behaviour. The thermo-chemical characteristics of stoichiometric methane-air flame are $s_{L}^{0}=0.4 \mathrm{~m} / \mathrm{s}, \delta_{L}^{0}=0.41 \mathrm{~mm}, \delta=0.047 \mathrm{~mm}$, $\tau=6.48$ and $\rho_{u} / \rho_{b}=7.54$.

The conditions of the three stoichiometric hydrogen-air flames used for comparative analyses are depicted in the combustion regime diagram shown in Fig. 3. These flames have the same stretch factor, $K=0.157\left(u^{\prime} / s_{L}^{0}\right)^{2} \operatorname{Re}_{t}^{-0.5}$ $=0.157$ [59], where the turbulent Reynolds number is defined as $\operatorname{Re}_{t}=u^{\prime} \Lambda / \nu$ with $\nu$ as the kinematic viscosity of the reactant mixture. For these flames, $u^{\prime} / s_{L}^{0}$ values are 5,6 and 8 and the stoichiometric methane-air flames also have the same combustion conditions as those for $\mathrm{H}_{2}$-air flames shown in Fig. 3. All of these flames are in the border between corrugated flamelets and thin reaction zones regime. The experimental flames of Kitagawa et al. [20] are also shown in this figure - the stoichiometric flame is near the border between wrinkled and corrugated flamelets while the lean flame with $\phi=0.4$ is near $\mathrm{Da}=1$ line, where $\mathrm{Da}=(\Lambda / \delta) /\left(u^{\prime} / s_{L}^{0}\right)$ is the Damköhler number.

\section{Results and discussion}

The computational results of spherically propagating hydrogen-air flames under a range of turbulence conditions are analysed in this section. Validation of the computational model is discussed first before presenting compar- 


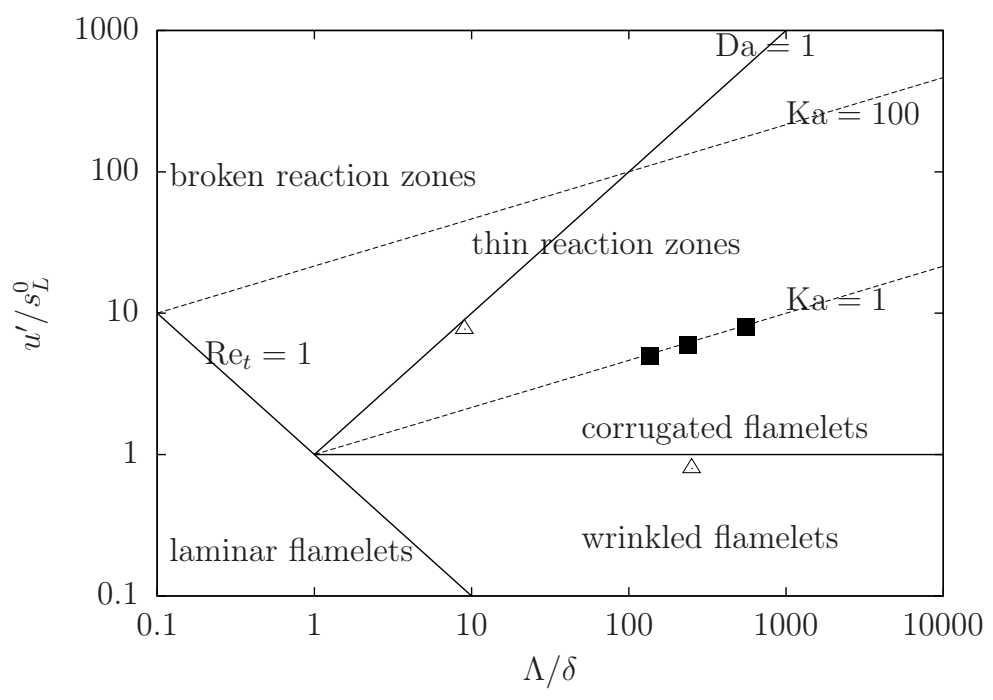

Fig. 3: Turbulent premixed combustion regime diagram [60] showing the conditions of test cases for further analyses ( $\mathbf{a})$ and the experimental case of Kitagawa et al. [20] $(\triangle)$ considered in this study.

ative analyses of hydrogen- and methane-air flames experiencing the same turbulent combustion conditions.

\subsection{Validation}

The amount of heat released by burning $m_{f}$ amount of fuel is simply given by $Q=m_{f} \mathrm{LHV}$, where $\mathrm{LHV}=120 \mathrm{MJ} / \mathrm{kg}$ is the lower heating value of hydrogen [5]. The amount of fuel consumed by the spherical turbulent flame when its leading edge moves from a radius of $r_{f 1}=4.2 \mathrm{~cm}$ to $r_{f 2}=7.74 \mathrm{~cm}$ over a period of $\Delta t=1.7 \mathrm{~ms}$ is $m_{f}=\rho_{u} Y_{f, u} 4 \pi\left(r_{f 2}^{3}-r_{f 1}^{3}\right) / 3$, where $Y_{f, u}=$ 0.028 is the fuel mass fraction in the stoichiometric hydrogen-air mixture. The leading edge radius, $r_{f}$, is defined using $\widetilde{c}=\widetilde{c}_{1}=0.05$ iso-level in the simulation and the above radii and $\Delta t$ are taken from one of the simulations chosen arbitrarily. The theoretical value for the heat release is $4.68 \mathrm{~kJ}$ and this value calculated from the results of numerical simulation using

$$
Q=4 \pi\left(T_{b}-T_{u}\right) \int_{t}^{t+\Delta t}\left[\int_{r_{f 1}}^{r_{f 2}} \bar{C}_{p}(r, t) \overline{\dot{\omega}}_{c_{T}}(r, t) r^{2} \mathrm{~d} r\right] \mathrm{d} t
$$


agrees within $6 \%$ of the above value. The symbol $\bar{C}_{p}(r, t)$ is the mean mixture heat capacity at constant pressure. This level of agreement is acceptable in the light of various approximations made in the modelling of turbulence and combustion.

As noted earlier, two atmospheric flames, $\phi=1$ and 0.4 , of Kitagawa et al. [20] are simulated to assess the strained flamelets model for hydrogen-air combustion. The variation of turbulent flame propagation speed, $u_{t}$, with $r_{f}$ was reported in [20] and this will be used for the validation purpose. This speed is defined as [61]:

$$
u_{t}=\frac{\bar{\rho}_{b}}{\rho_{u}} \frac{\mathrm{d} r_{f}}{\mathrm{~d} t}
$$

where $\bar{\rho}_{b}$ is the mean density of the burnt gases. The numerical simulations give $\widetilde{c}(r, t)$ (see figure 5 , to be discussed later) and thus it is straightforward to compute $u_{t}$. Figure 4 compares the computed and measured $u_{t}$ variation with $r_{f}$ for the stoichiometric, Fig. 4a, and lean, Fig. 4b, hydrogen-air flames. The computational results are shown for three combustion models described in Section 3. The chemical kinetics mechanism of Li et al. [37] is used for the unstrained flamelets model in Eq. (4). The mechanisms of both Li et al. [37] (noted as mech 1 in Fig. 4a) and Westbrook [34] (mech 2) are used for the strained flamelets model in order to assess the influence of chemical kinetics. Both the algebraic and the unstrained flamelet models yield larger $u_{t}$ values.

The strained flamelet model with Li et al.'s [37] mechanism is able to capture the measured variation reasonably well, whereas the values of $u_{t}$ computed using Westbrook's [34] mechanism are smaller as in Fig. 4a. This is because the mechanism of Westbrook [34] underpredicts the laminar burning velocity, $s_{L}^{0}$, as was shown in Section 2.1 (see Fig. 1). This highlights the importance of choosing a chemical mechanism that gives accurate laminar flame characteristics required for the strained flamelets model. The maximum error between the measured and computed $u_{t}$ values is about $5 \%$ for the strained flamelets model and this error is well within the experimental scatter of Kitagawa et al. [20]. However, a close study of Fig. 4a shows that there is a substantial difference between the measured and computed $u_{t}$ values for $r_{f}<10 \mathrm{~mm}$. When the flame radius is smaller than the turbulence integral length scale (about $10 \mathrm{~mm}$ for this experiment as noted earlier), the flame will be simply convected by the large-scale motion and the interaction between the flame and turbulence is limited to a very small part of a wide 


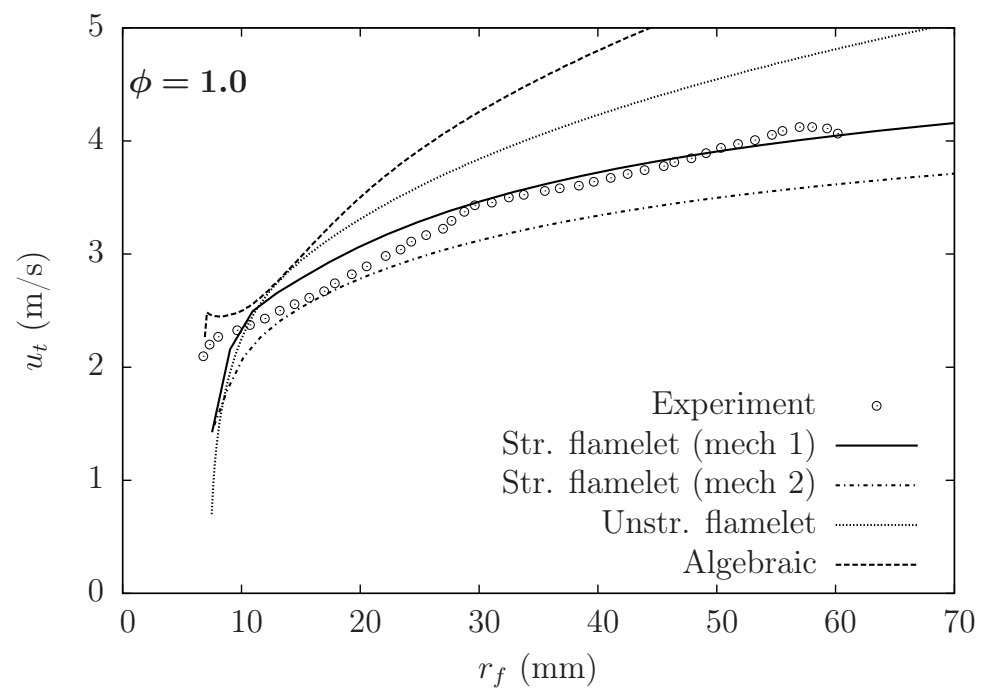

(a)

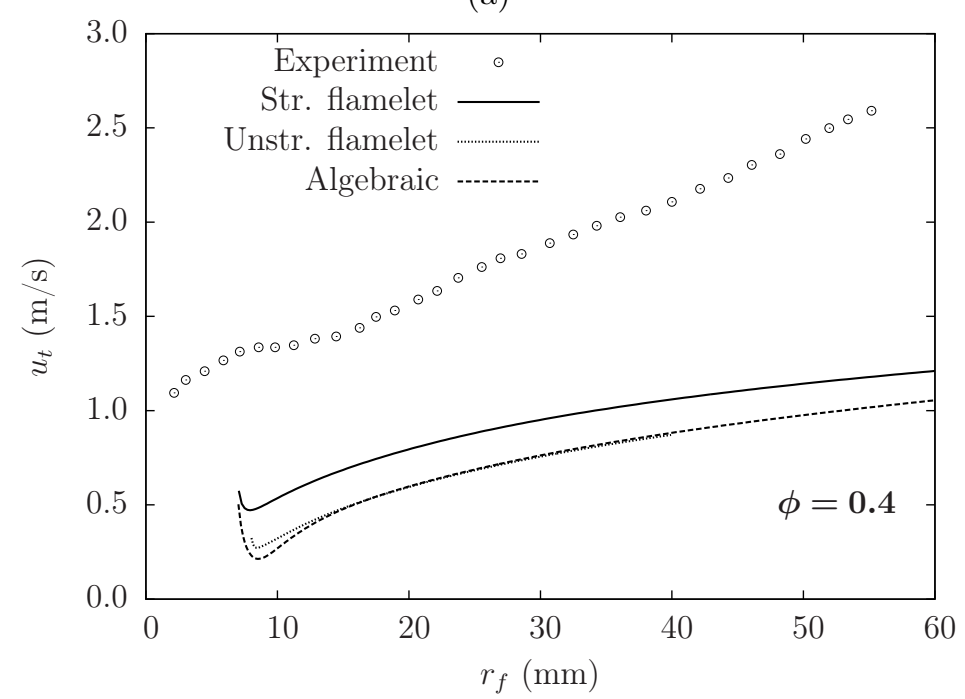

(b)

Fig. 4: Comparison of computed and measured variation of $u_{t}$ with $r_{f}$ in (a) stoichiometric and (b) lean $(\phi=0.4)$ hydrogen-air flames. 
spectrum of turbulence scales and so, the RANS combustion modelling may not hold as noted in earlier studies [33,62]. One must be cautious to interpret RANS results when $r_{f} \leq \Lambda$.

The variation of $u_{t}$ shown in Fig. 4 suggests that the burning velocity for the spherical flame is not constant as has been observed in many earlier studies $[20,23,24]$. In turbulent flames, the mean reaction rate depends on the fuel, the equivalence ratio and local turbulent strain rate [63]. In these flames, local fluid dynamic strain induced by turbulent eddies acts to reduce the burning rate. Amongst the models used in this study, the strained flamelets model seem to describe both the complex chemistry and the local fluid dynamic effects quite well for stoichiometric hydrogen-air flame. This flame is thermo-diffusively stable whereas the lean flame having $\phi=0.4$ is thermo-diffusively unstable as noted by Kitagawa et al. [20]. Figure 4b compares the measured and computed $u_{t}$ values for $\phi=0.4$ flame. All the three models severely under estimate the turbulent burning velocity because these models do not include the influences of thermo-diffusive instability on the propagation of turbulent flame leading edge. Including these effects in turbulent combustion modelling is a challenging task and one way to include them may be through the use of Markstein numbers but this approach is strictly valid for small stretch rates $\kappa^{+} \equiv \kappa \delta_{L}^{0} / s_{L}^{0} \ll 1$ [64]. In the remainder of this paper only stoichiometric hydrogen-air flames will be considered for further and comparative analyses.

\subsection{Comparison of hydrogen- and methane-air flames}

\subsubsection{Propagation characteristics}

The stoichiometric hydrogen- and methane-air spherical flames experiencing the same combustion conditions are compared in this section. Typical evolution of these flames is shown in Fig. 5 for $u^{\prime} / s_{L}^{0}=6$ case. Both of these flames evolve from the same initial variation of $\widetilde{c}$ shown as dashed lines and the results are shown for a period of $3 \mathrm{~ms}$ at an equal interval of $1 \mathrm{~ms}$. The symbols used for $3 \mathrm{~ms}$ indicates typical spatial resolution used in simulations. The stoichiometric hydrogen flames propagate faster than the methane flames, which is expected since the laminar burning velocity for the hydrogen-air mixture is larger than for the methane-air mixture.

Variation of flame radius, normalised by its initial value, with time is shown in Fig. 6 for all $6\left(3 \mathrm{H}_{2^{-}}\right.$and $3 \mathrm{CH}_{4}$-air $)$ flames considered in this study. For a given combustion condition denoted by $u^{\prime} / s_{L}^{0}$ and $\Lambda / \delta$, the hydrogen flames propagate significantly, nearly 4 to 5 times, faster compared 

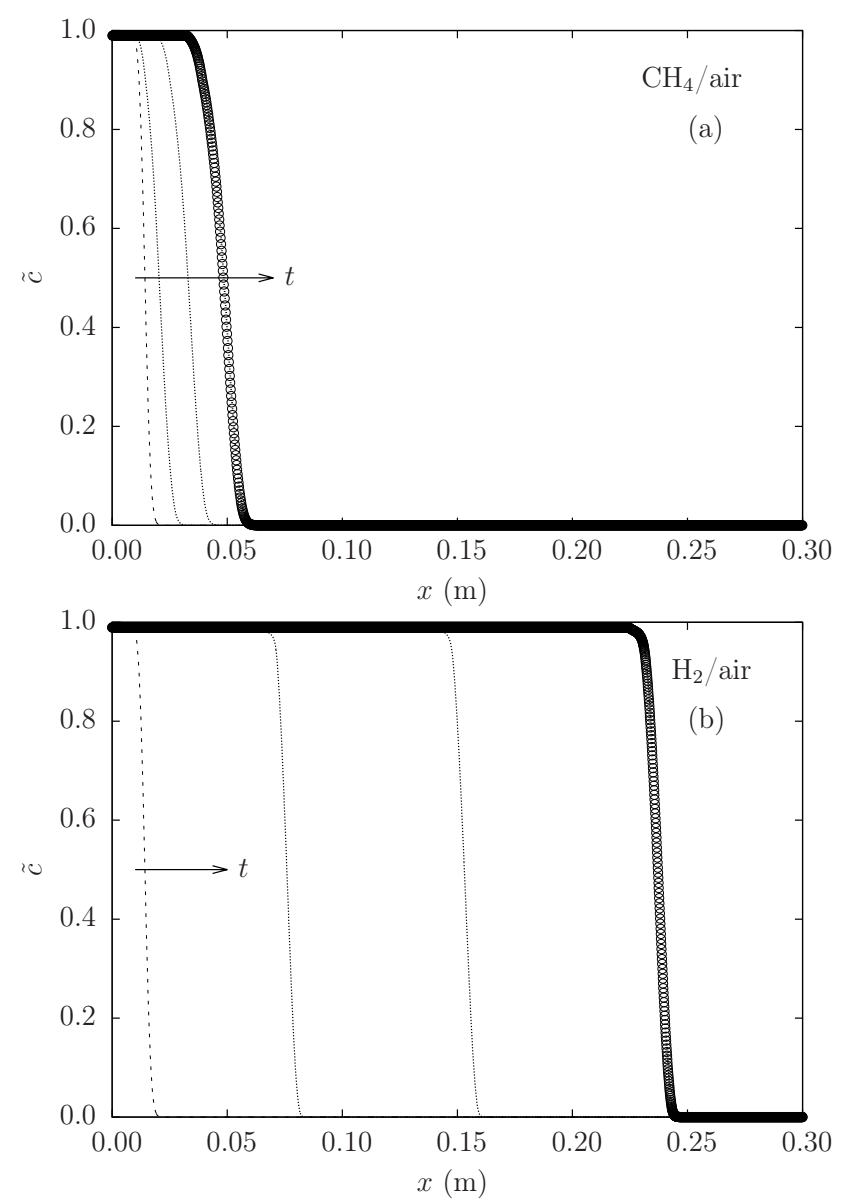

Fig. 5: Spatial variation of $\widetilde{c}$ at four instances from 0 to $3 \mathrm{~ms}$ at an interval of $1 \mathrm{~ms}$ in (a) $\mathrm{CH}_{4}$-air and (b) $\mathrm{H}_{2}$-air flames having $u^{\prime} / s_{L}^{0}=6$. 
to the methane-air flames. This result also shows that the increase in $u^{\prime}$, for a constant $K$ or Ka, results in faster flame propagation for both of these fuel-air mixtures. This increase in flame propagation speed agrees with many previous studies summarised in [65].

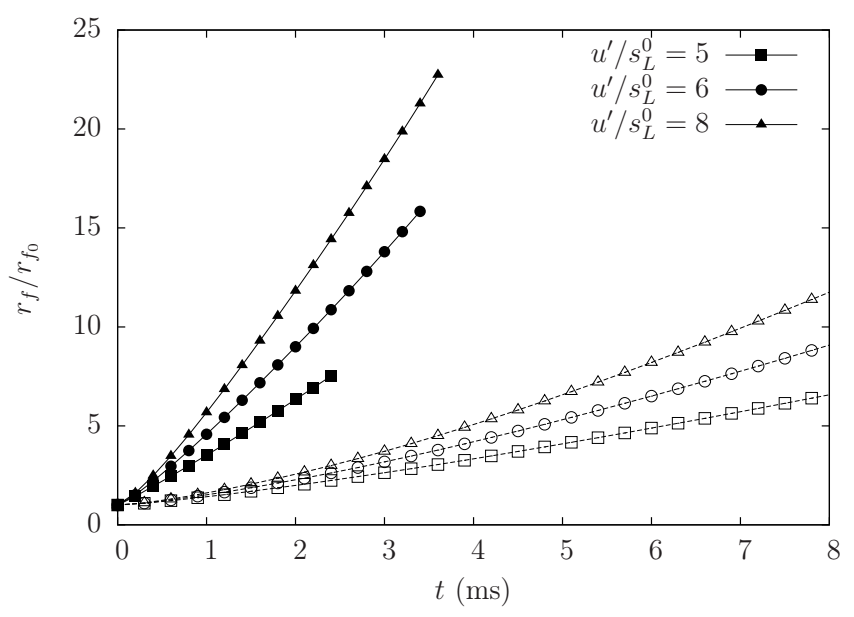

Fig. 6: Temporal variation of normalised flame radius of $\mathrm{CH}_{4}$-air (open symbols) and $\mathrm{H}_{2}$-air (closed) turbulent flames at various combustion conditions.

\subsubsection{Turbulent flame speed and consumption speed}

The turbulent flame speed, $s_{t}$, is of interest to theoretical and practical investigations of turbulent premixed flames. Unlike the laminar flame speed, $s_{t}$ depends on flame geometry as well as mixture properties [66]. The flame speed is defined to be the displacement speed of the flame brush leading edge marked using $\widetilde{c}=0.05$ in this study. The displacement speed given by $s_{d}=\left[\mathrm{d} \boldsymbol{r}_{f} / \mathrm{d} t\right] \cdot \boldsymbol{n}-\widetilde{u} \cdot \boldsymbol{n}$, where $\widetilde{u}$ is the Favre averaged fluid velocity and $\boldsymbol{n}$ is a unit normal vector to the iso-surface of $\widetilde{c}=0.05$. It is also well known that this displacement speed is influenced directly by the mean reaction rate, turbulent flux and molecular diffusion [33, 60, 64]. Note the difference in definitions of $s_{t}$ and the turbulent burning velocity, $u_{t}$, given in Eq. (9).

Figure 7a shows the variation of normalised turbulent flame speed, $s_{t} / s_{L}^{0}$, with turbulent Reynolds number, $\mathrm{Re}_{t}$. The results are shown for a normalised time of $t^{+}=8$ and the results for other earlier times are similar to those shown here as noted in [33] for hydrocarbon flames. The inset shows the 
variation of $s_{t} / s_{L}^{0}$ with $u^{\prime} / s_{L}^{0}$, which suggests that $s_{t} \sim u^{\prime}$ as noted earlier by Bray [67]. Lipatnikov and Chomiak [65] analysed a number of experimental flames to show that $s_{t} \sim u^{\prime q}$, with $0.5 \leq q \leq 1$. This increase in $s_{t}$ with $u^{\prime}$ is generally believed due to turbulent eddies increasing the flame surface area by stretching and contorting it [65]. The slopes of $s_{t} / s_{L}^{0}$ versus $u^{\prime} / s_{L}^{0}$ curves are 6.3 and 4.4 respectively for the methane- and hydrogen-air flames considered here. It was observed that this slope became steeper when the stretch factor, $K$, is increased in an earlier study [33].

The results also suggest a relation, $s_{t} / s_{L}^{0} \simeq B \mathrm{Re}_{t}^{n}$ for both flames with $n \approx 0.5$. The least squares fit shown in the figure suggests $B=0.21$ for the methane- and 0.16 for hydrogen-air flames. The approximate square root dependence of $s_{t}$ on $\mathrm{Re}_{t}$ is consistent with the classical analysis of Damköhler for thin reaction zones combustion using a hypothesis, $s_{t} \sim \sqrt{D_{t} / t_{c}}$, where $D_{t}$ is the turbulent diffusivity. This is analogous to $s_{L}^{0} \sim \sqrt{D / t_{c}}$, where $D$ is the molecular diffusivity, in the laminar flame theory [60]. Chaudhuri et al. [68] observed a similar square-root dependence for spherical and Bunsen methane-air flames that are thermo-diffusively and hydrodynamically stable. These results suggest that this scaling relation is applicable to both hydrogen and hydrocarbon flames.

The variation of consumption speed, $s_{c}$, defined as $\rho_{u} s_{c}=\int_{0}^{1}(\overline{\dot{\omega}} /|\partial \widetilde{c} / \partial r|) \mathrm{d} \widetilde{c}$, with $\mathrm{Re}_{t}$ is shown in Fig. 7b. The scaling of $s_{c} / s_{L}^{0}$ with $\operatorname{Re}_{t}$ is similar to that of $s_{t} / s_{L}^{0}$. However, the magnitude of $s_{c}$ is smaller than $s_{t}$. As with $s_{t} / s_{L}^{0}$, the computed values of $s_{c} / s_{L}^{0}$ are about $20 \%$ lower for hydrogen-air flames when compared with methane-air flames, which is because of larger $s_{L}^{0}$ value.

\subsubsection{Turbulent flame brush thickness}

Figure 8 shows the temporal variation of flame brush thickness for the stoichiometric $\mathrm{H}_{2}$-air flame with $u^{\prime} / s_{L}^{0}=8$. The flame brush thickness is normalised using the laminar flame thermal thickness and the time is normalised using the flame time, $t_{c}=\delta_{L}^{0} / s_{L}^{0}$. The flame brush thickness is defined in two ways, one is using the maximum gradient of $\widetilde{c}$ and another one is using the variance $\sigma_{c}^{2}$. This second thickness is defined as the thickness over which $\sigma_{c}^{2}$ falls to $5 \%$ of its maximum value to be consistent with Taylor's theory of turbulent dispersion [69]. Note that the variance thickness is also scaled to fit in the scale shown in Fig. 8. After going through some initial transients, both thickness grow with time. If the turbulent diffusion plays the central role for this growth then one would expect to see a growth similar to that shown for Taylor's theory. This theory suggests a linear variation for 

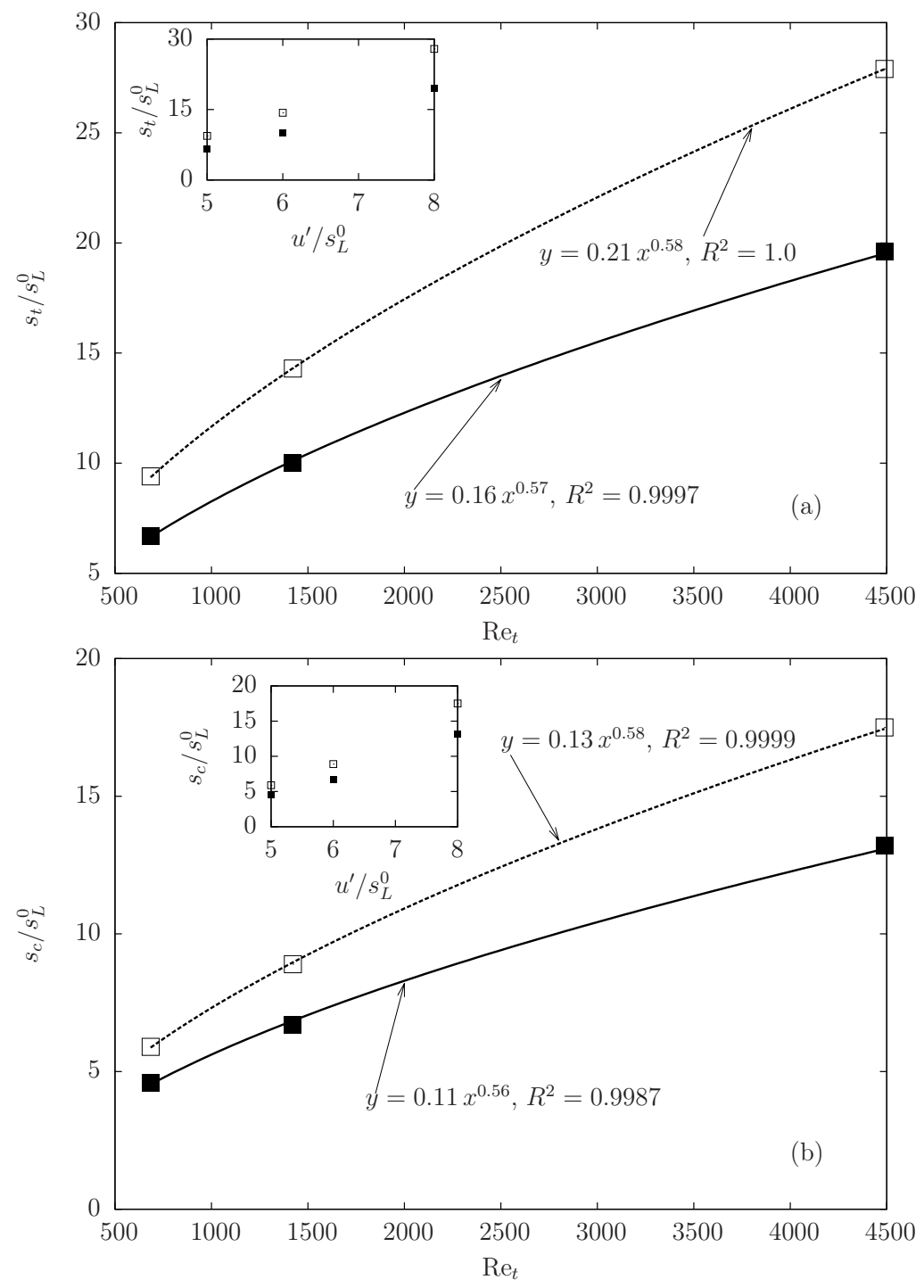

Fig. 7: Variation of normalised turbulent (a) flame and (b) consumption speeds of $\mathrm{H}_{2}$-air (closed symbols) and $\mathrm{CH}_{4}$-air flames (open) with turbulence Reynolds number. The inset shows the variations with $u^{\prime}$. The results are shown for $t^{+}=8$. 
$t^{+} \ll$ Da and a square root dependence for $t^{+} \gg$ Da. It is apparent that the computed thicknesses do not follow these variations as it has been observed for methane-air flames [33]. Thus, it is concluded here the growth of the flame brush thickness in the stoichiometric hydrogen-air flames studied here is governed by the differential propagation of the leading and trailing edges of the flame brush as for the methane-air flames [33]. The leading edge propagates faster compared to the trailing edge.

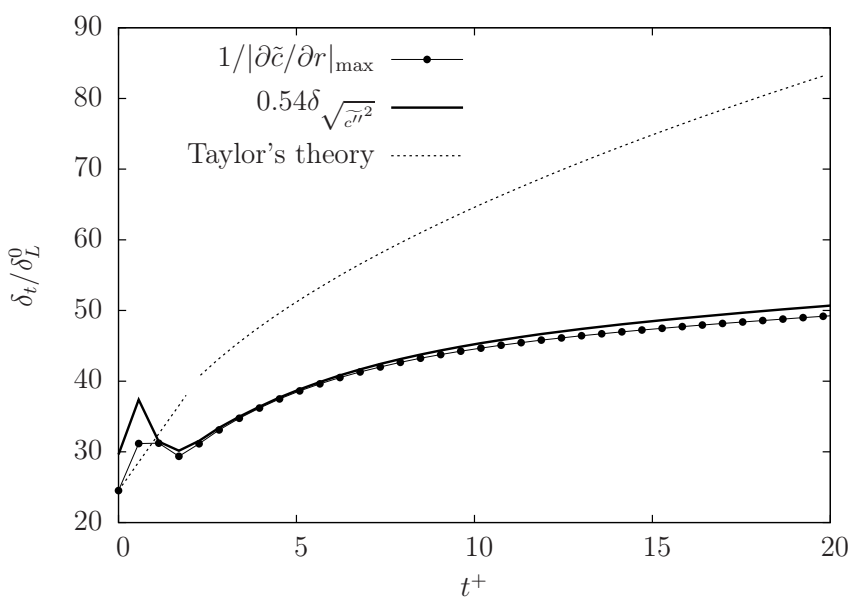

Fig. 8: Temporal variation of normalised flame brush thickness for $\mathrm{H}_{2}$-air flame with $u^{\prime} / s_{L}^{0}=8$.

According to the concept of turbulent premixed combustion regimes, two flames with identical values for $\Lambda / \delta, u^{\prime} / s_{L}^{0}$ and $\tau$ are expected to have similar $s_{t} / s_{L}^{0}$. Although these quantities are kept to be identical for the hydrogenand methane-air flames investigated in this work, there is a significant difference in $s_{t} / s_{L}^{0}$. The well known KPP analysis [70] shows that $s_{t}$ strongly depends on the rate of change of $\overline{\dot{\omega}}_{c}$ with respect to $\widetilde{c}$ as $\widetilde{c} \rightarrow 0$ and this quantity not only depends on the turbulence-chemistry interaction but also on the combustion kinetics. The turbulence-chemistry interaction is expected to be predominantly the same if $u^{\prime} / s_{L}^{0}$ and $\Lambda / \delta$ are kept the same. To gain an understanding of $\left(\partial \overline{\dot{\omega}}_{c} / \partial \widetilde{c}\right)_{\widetilde{c} \rightarrow 0}$, the variation of $\overline{\dot{\omega}}_{c}$ with $\widetilde{c}$ is studied next.

\subsubsection{Mean reaction rates}

Figure 9 shows mean reaction rate variation across the flame brush of hydrogen- and methane-air flames. The mean reaction rates are normalised 
by the respective maximum value inside the flame brush. The top inset shows the maximum value of the normalised mean reaction rate, $\overline{\dot{\omega}}_{\text {max }}^{+}$, for different turbulence conditions considered for this study. These results show that turbulence level does not have a significant effect on $\overline{\dot{\omega}}_{\text {max }}^{+}$, which agrees with recent direct numerical simulation results [71]. However, there is some difference in the variation of normalised reaction rate across the flame brush between the methane- and hydrogen-air mixtures. The mean reaction rate is more uniform inside the flame brush for hydrogen compared to methane. The difference between these two flames looks small for low $\widetilde{c}$ values, however the bottom inset clearly shows that there is a substantial difference. The value of $\left(\partial \overline{\dot{\omega}}_{c} / \partial \widetilde{c}\right)_{\widetilde{c} \rightarrow 0}$ for the hydrogen flame can be two orders of magnitude larger than for the methane flame. This is because of the low activation temperature for hydrogen combustion. Obviously, this parameter is related to chemical kinetics of the fuel.

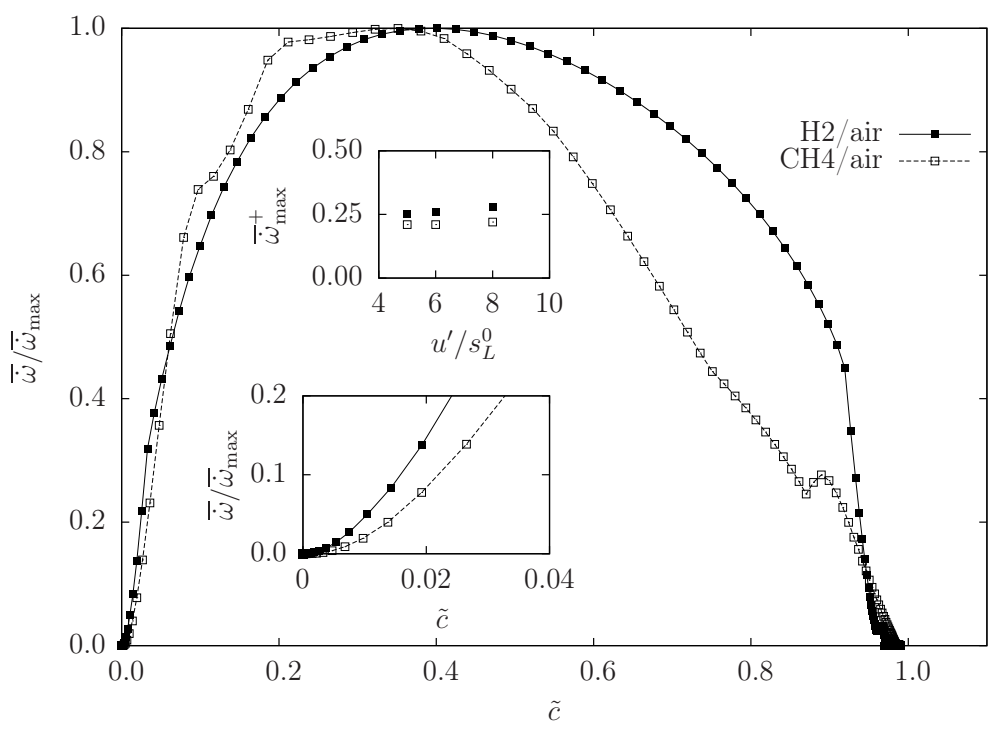

Fig. 9: Variation of normalised mean reaction rate with $\widetilde{c}$ for both $\mathrm{H}_{2}$ - and $\mathrm{CH}_{4}$-air flames for $u^{\prime} / s_{L}^{0}=6$ at $t^{+}=8$. The top inset shows the variation of $\overline{\dot{\omega}}_{\text {max }}^{+}$, normalised as $\overline{\dot{\omega}}_{\max } \delta_{L}^{0} /\left(\rho_{u} s_{L}^{0}\right)$, with $u^{\prime} / s_{L}^{0}$. 


\section{Conclusions}

Numerical simulations of turbulent spherical hydrogen-air flames have been carried out using RANS methodology employing three different reaction rate closures: an algebraic model of Bray [53], strained and unstrained flamelets. Since the hydrogen-air mixture has non-unity Lewis number, a two progress variable approach is used in this work to account for differential evolution of temperature and mass fraction in hydrogen-air combustion. This modelling approach is assessed using the experimental data of Kitagawa et al. [20]. The measured variation of propagation speed of flame brush leading edge with flame radius is compared with the computed values for stoichiometric and a lean, $\phi=0.4$, flames. This comparison demonstrate that the stretch effects on flamelets must be included to capture experimental measurements. The strained flamelets model is able to capture the experimental variations quite well while the unstrained flamelets and the algebraic models give faster flame propagation. Also, the results showed that the chemical kinetics mechanism to be used in the calculation must capture the laminar flame characteristics such burning velocity, flame thermal thickness, flame structure, etc., of the corresponding mixture well. The use of the strained flamelets modelling approach is justified for stoichiometric hydrogen-air flames as these flames are thermo-diffusively stable. The propagation speed of the lean flame, which is thermo-diffusively unstable, is under estimated by all the three combustion models used in this study. It seems that the approach of including the thermo-diffusive effects in the laminar flamelets is inadequate and an alternative methodology need to be found.

The results of stoichiometric hydrogen- and methane-air spherical flames obtained using strained flamelets model are analysed comparatively to understand the relative effects of turbulence on the propagation of these flames. It is observed that $s_{t} \sim u^{\prime}$ for a constant value of turbulence stretch rate for both hydrogen- and methane-air flames. Furthermore, the normalised turbulent flame speed, $s_{t} / s_{L}^{0}$, and consumption speed, $s_{c} / s_{L}^{0}$, scale as $\operatorname{Re}_{t}^{n}$, with $n \approx 0.5$ for both mixtures. However, the magnitudes of these speeds are observed to be substantially different for the stoichiometric hydrogen- and methane-air mixtures despite the fact that these $\mathrm{H}_{2}$ - and $\mathrm{CH}_{4}$-air flames have identical combustion conditions in terms of $u^{\prime} / s_{L}^{0}$ and $\Lambda / \delta$, implying similar turbulence-flame interactions. It is observed that this difference is related to behaviour of $\left(\partial \overline{\dot{\omega}}_{c} / \partial \widetilde{c}\right)_{\widetilde{c} \rightarrow 0}$, which is controlled not only by turbulence and its interaction with the flame but also by chemical kinetics. This gradient 
value is observed to be nearly two orders of magnitude larger for the $\mathrm{H}_{2}$-air flame compared to the $\mathrm{CH}_{4}$-air flame. The predominant role of differential propagation between the leading and trailing edges of the flame brush on the growth of the flame brush thickness is also observed for the hydrogen spherical flames.

\section{References}

[1] M. A. DeLuchi, Int. J. Hydrogen Energ. 14 (1989) 81-130.

[2] J. M. Ogden, Annu. Rev. Energ. Env. 24 (1999) 227-279.

[3] M. Balat, Int. J. Hydrogen Energ. 33 (2008) 4013-4029.

[4] C. M. White, R. R. Steeper, A. E. Lutz, Int. J. Hydrogen Energ. 31 (2006) 1292-1305.

[5] S. Verhelst, T. Wallner, Prog. Energ. Combust. 35 (2009) 490-527.

[6] C. G. Bauer, T. W. Forest, Int. J. Hydrogen Energ. 26 (2001) 55-70.

[7] A. Stohl, P. Seibert, G. Wotawa, D. Arnold, J. F. Burkhart, S. Eckhardt, C. Tapia, A. Vargas, T. J. Ysunari, Atmos. Chem. Phys. 12 (2012) 23132343.

[8] R. K. Kumar, W. A. Dewit, D. R. Greig, Combust. Sci. and Technol 66 (1989) 251-266.

[9] V. V. Molkov, D. V. Makarov, H. Schneider, Int. J. Hydrogen Energ. 32 (2007) 2198-2205.

[10] C. K. Law, C. J. Sung, Prog. Energ. Combust. 26 (2000) 459-505.

[11] D. R. Dowdy, D. B. Smith, S. C. Taylor, A. Williams, Proc. Combust. Inst. 23 (1990) 325-332.

[12] S. Kwon, L. K. Tseng, G. M. Faeth, Combust. Flame 90 (1992) 230-246.

[13] K. T. Aung, M. I. Hassan, G. M. Faeth, Combust. Flame 112 (1998) $1-15$.

[14] O. C. Kwon, G. M. Faeth, Combust. Flame 124 (2001) 590-610. 
[15] S. D. Tse, D. L. Zhu, C. K. Law, Proc. Combust. Inst. 28 (2000) 17931800 .

[16] O. C. Kwon, G. Rozenchan, C. K. Law, Proc. Combust. Inst. 29 (2002) 1775-1783.

[17] S. Verhelst, A study of combustion in hydrogen-fuelled internal combustion engines, Ph.D., Ghent University, Ghent, Belgium, 2005.

[18] S. Verhelst, R. Woolley, M. Lawes, R. Sierens, Proc. Combust. Inst. 30 (2005).

[19] D. Bradley, M. Lawes, K. Liu, S. Verhelst, R. Woolley, Combust. Flame 149 (2007) 162-172.

[20] T. Kitagawa, T. Nakahara, K. Maruyama, K. Kado, A. Hayakawa, S. Kobayashi, Int. J. Hydrogen Energ. 33 (2008) 5842-5849.

[21] M. Matalon, Annu. Rev. Fluid. Mech. 39 (2007) 163-191.

[22] M. S. Wu, S. Kwon, J. F. Driscoll, G. M. Faeth, Combust. Sci. Technol. 73 (1990) 327-350.

[23] S. Kwon, M. S. Wu, J. F. Driscoll, G. M. Faeth, Combust. Flame 88 (1992) 221-238.

[24] K. T. Aung, M. I. Hassan, S. Kwon, L. K. Tseng, O. C. Kwon, G. M. Faeth, Combust. Sci. Technol. 174 (2002) 61-99.

[25] H. Kido, M. Nakahara, K. Nakashima, J. Hashimoto, Proc. Combust. Inst. 29 (2002) 1855-1861.

[26] B. Renou, A. Boukhalfa, D. Puechberty, M. Trinité, Proc. Combust. Inst. 27 (1998) 841-847.

[27] B. Renou, A. Boukhalfa, D. Puechberty, M. Trinité, Combust. Flame 123 (2000) 507-521.

[28] R. N. Paul, K. N. C. Bray, in: Proc. Combust. Inst., volume 26, pp. 259-266.

[29] S. P. R. Muppala, M. Nakahara, N. K. Aluri, H. Kido, J. X. Wen, M. V. Papalexandris, Int. J. Hydrogen Energ. 34 (2009) 9258-9265. 
[30] U. Gerke, Numerical analysis of mixture formation and combustion in a hydrogen direct-injection internal combustion engine, Ph.D., ETH Zurich, No. 17477, Cuvillier Göttingen, Switzerland, 2007.

[31] C. D. Rakopoulos, G. M. Kosmadakis, E. G. Pariotis, Int. J. Hydrogen Energ. 35 (2010) 12545-12560.

[32] H. Kolla, N. Swaminathan, Combust. Flame 157 (2010) 943-954.

[33] I. Ahmed, N. Swaminathan, Combust. Sci. Technol. 185 (2013) 15091540 .

[34] C. K. Westbrook, Combust. Sci. Technol. 29 (1982) 67-81.

[35] J. A. Miller, Proc. Combust. Inst. 19 (1982) 181-196.

[36] M. Ó Conaire, H. J. Curran, J. M. Simmie, W. J. Pitz, C. K. Westbrook, Int. J. Chem. Kinet. 36 (2004) 603-622.

[37] J. Li, Z. Zhao, A. Kazakov, F. L. Dryer, Int. J. Chem. Kinet. 36 (2004) 566-575.

[38] M. P. Burke, M. Chaos, Y. Ju, F. L. Dryer, S. J. Klippenstein, Int. J. Chem. Kinet. 44 (2011) 444-474.

[39] C. K. Wu, C. K. Law, Proc. Combust. Inst. 20 (1984) 1941-1949.

[40] F. N. Egolfopoulos, C. K. Law, Proc. Combust. Inst. 23 (1990) 333-340.

[41] C. M. Vagelopoulos, F. N. Egolfopoulos, C. K. Law, Proc. Combust. Inst. 25 (1994) 1341-1347.

[42] K. T. Aung, M. I. Hassan, G. M. Faeth, Combust. Flame 109 (1997) $1-24$.

[43] N. Lamoureux, N. Djebali-Chaumeix, C. E. Paillard, Exp. Therm. Fluid Sci. 27 (2003) 385-393.

[44] Z. Huang, Y. Zhang, K. Zeng, B. Liu, Q. Wang, D. Jiang, Combust. Flame 146 (2006) 302-311.

[45] C. Tang, Z. Huang, C. Jin, J. He, J. Wang, X. Wang, H. Miao, Int. J. Hydrogen Energ. (2008) 4906-4914. 
[46] E. Hu, Z. Huang, J. He, J. Zheng, H. Miao, Int. J. Hydrogen Energ. 34 (2009) 5574-5584.

[47] J. Pareja, H. J. Burbano, Y. Ogami, Int. J. Hydrogen Energ. 35 (2010) 1812-1818.

[48] M. Kuznetsov, S. Kobelt, J. Grune, T. Jordan, Int. J. Hydrogen Energ. 37 (2012) 17580-17588.

[49] N. Chakraborty, N. Champion, A. Mura, N. Swaminathan, in: N. Swaminathan, K. N. C. Bray (Eds.), Turbulent Premixed Flames, Cambridge University Press, Cambridge, UK, 2011, pp. 74-102.

[50] H. Kolla, J. W. Rogerson, N. Chakraborty, N. Swaminathan, Combust. Sci. Technol. 181 (2009) 518-535.

[51] J. W. Rogerson, N. Swaminathan, European Combustion Meeting (2007).

[52] H. Kolla, N. Swaminathan, J. Combust. 2011 (2011) 1-8.

[53] K. N. C. Bray, Proc. Combust. Inst. 17 (1979) 223-233.

[54] Y. Minamoto, N. Fukushima, M. Tanahashi, T. Miyauchi, T. D. Dunstan, N. Swaminathan, Phys. Fluids 23 (2011).

[55] R. J. Kee, J. F. Grcar, M. Smooke, J. A. Miller, PREMIX: A Fortran program for modeling steady one-dimensional flames, Sandia National Laboratories, 1985. SAND85-8240.

[56] A. E. Lutz, R. J. Kee, J. F. Grcar, F. M. Rupley, OPPDIF: A FORTRAN program for computing opposed-flow diffusion flames, Sandia National Laboratories, 1997. Tech. Rep. SAND96-8243.

[57] S. V. Patankar, Numerical Heat Transfer and Fluid Flow, Taylor and Francis, London, 1980.

[58] D. Bradley, P. H. Gaskell, X. J. Gu, Combust. Flame 104 (1996) 176198.

[59] R. G. Abdel-Gayed, D. Bradley, M. Lawes, Proc. R. Soc. Lond. A 414 (1987) 389-413. 
[60] N. Peters, J. Fluid Mech. 384 (1999) 107-132.

[61] G. E. Andrews, D. Bradley, Combust. Flame 19 (1972) 275-288.

[62] S. B. Pope, Ann. Rev. Fluid Mech. 19 (1987) 237-270.

[63] R. S. Cant, K. N. C. Bray, Combust. Flame 76 (1989) 243-263.

[64] T. Poinsot, D. Veynante, Theoretical and numerical combustion, Edwards, Philadelphia, 2nd edition, 2005.

[65] A. Lipatnikov, J. Chomiak, Prog. Energ. Combust. 28 (2002) 1-74.

[66] J. F. Driscoll, Prog. Energ. Combust. 34 (2008) 91-134.

[67] K. N. C. Bray, Proc. R. Soc. Lond. A 431 (1990) 315-335.

[68] S. Chaudhuri, F. Wu, D. Zhu, C. K. Law, Phys. Rev. Lett. 108 (2012).

[69] G. Taylor, Proc. R. Soc. Lond. A 151 (1935) 421-444.

[70] Y. Zel'dovich, G. Barenblatt, V. Librovich, G. Makhviladze, The Mathematical Theory of Combustion and Explosions, Consultants Bureau, New York, 1985.

[71] T. D. Dunstan, N. Swaminathan, K. N. C. Bray, J. Fluid Mech. 709 (2012) 191-222. 